\title{
Are Heterogeneous Cellular Networks Superior to Homogeneous Ones?*
}

\author{
Shelly Salim, Christian H.W. Oey, and Sangman Moh ${ }^{* *}$ \\ Dept. of Computer Engineering, Chosun University, Gwangju, South Korea \\ smmohachosun.ac. kr
}

\begin{abstract}
In this paper, the performance of homogeneous cellular networks (HMCNs) and heterogeneous cellular networks (HTCNs) is evaluated and compared. The HTCN discussed in this paper consists of three kinds of cells: macrocells, microcells and femtocells. The macrocells are evenly deployed. The microcells are densely deployed in the offices and public areas and sparsely deployed in the universities areas. The femtocells are deployed in the residential areas. And, a user mobility pattern is defined to model real communication environment. Our simulation results show that the HTCN requires less power (in Watt $/ \mathrm{km}^{2} / \mathrm{Mbps}$ ) and achieves higher throughput compared to the HMCN.
\end{abstract}

Keywords: Heterogeneous cellular network, user mobility, energy, throughput.

\section{Introduction}

There are rising concerns about energy conservation in information and communication technology (ICT) field. ICT contributes approximately $2 \%$ of global carbon emission and it is predicted to reach $2.8 \%$ by 2020 [1]. Among ICTs, the base stations of cellular mobile networks are the most energy consuming component that consumes about an average of $25 \mathrm{MWh}$ per year. Millions of base stations are deployed and they will be increased in developing regions. Therefore, the efforts to suppress base stations' power consumption are desperately needed. Besides environment friendly aspect, reducing power consumption of the base stations would also advantage the network operators by lowering the operators' expenditure. The efforts to reduce the power consumption of cellular networks coined the trendy term "green cellular network".

The continuous demand of higher data rate is considered to be the main reason of increasing energy consumption in cellular networks. Users' traffic growth could reach $400 \%$ per year, for both data and voice traffic [2]. Therefore, on one hand, the service providers need to reduce the energy consumption of the cellular network, but on the other hand, they also have to meet the demand for higher data rate.

\footnotetext{
* This work was supported in part by the MKE (The Ministry of Knowledge Economy), Korea, under the ITRC (Information Technology Research Center) support program supervised by the NIPA (National IT Industry Promotion Agency) (NIPA-2012-H0301-12-2008).

** Corresponding author.
} 
Various strategies have been proposed to reduce power consumption in cellular networks. In [3], two approaches are mentioned; one is by utilizing energy-aware components in the base stations and the other is by employing energy-aware network deployment. Detailed discussions are provided in a survey paper [4], which categorizes the energy saving solutions into architectural, network planning, and system design approaches. The architectural approach focuses on energy saving in the base stations, such as cooperative base stations and renewable energy sources implementation. New technologies, such as cognitive radio and cooperative relays, are considered in the system design approach, in addition to energy-aware communication protocols, resource management, cross-layer design, etc. This paper is especially attracted to the network planning approach, which is about the deployment of heterogeneous cellular networks (HTCN), since it is a promising candidate to achieve both energy conservation and higher data rates.

The straightforward method to increase power efficiency is by decreasing the propagation distance, since it will reduce the transmission power. Therefore, the deployment of smaller cells forming a heterogeneous network is a promising solution. The common base stations of large area coverage, called macrocells, are mainly designed to cover a large transmission area rather than to provide high data rates. While the base stations of small area coverage, called microcells, are low power base stations capable to handle dense traffic. Also, smaller cells could utilize higher frequency bands, allowing them to support high data rates. Moreover, smaller cells encourage spectral efficiency by increasing spatial frequency reuse. In this paper, HTCN consisting of macrocells, microcells and femtocells is introduced with a user mobility pattern and it is compared to the homogeneous cellular network (HMCN) of macrocells only in terms of energy efficiency and system throughput.

The rest of this paper is organized as follows: Some related works are summarized in the following section. A HTCN is presented as a design under test with a user mobility pattern. The performance of the HTCN is evaluated and compared in Section 4. Finally, conclusions are covered in Section 5.

\section{Related Works}

To the best of our knowledge, the works on HTCNs consider either joint deployment of macrocells and microcells (picocells) or joint deployment of macrocells and femtocells, without the consideration of the three of them deployed simultaneously. In this paper, the HTCN consists of macrocells, microcells and femtocells. Also, a user mobility pattern is included to model real mobile communication environment.

There are many works proposed in the field of cellular networks energy conservation. Some of them concentrate on the deployment of heterogeneous cellular networks or small scale cellular networks. The work in [5] studied the energy efficiency of joint macrocells and picocells network. It concluded that joint deployment strategy could reduce the total energy consumption in urban areas by up to $60 \%$.

The energy efficiency of cellular networks with femtocells is studied in [6]. It presented an energy consumption modeling framework where the base stations have 
three states, those are: 'turn-off state', 'radio-off state' and 'radio-on state'. Each state represents different energy consumption. The simulation results in [6] showed that as femtocells penetration rate is increased, the normalized energy consumption per cell is decreased; as the number of open access mobile nodes is increased, the system throughput is increased. In [7], simulation-based case studies of macrocells offloading benefits in UMTS (Universal Mobile Telecommunications System) and WiMAX (Worldwide Interoperability for Microwave Access) networks are presented. Both results support femtocells deployment in macrocells. However, those works stated above excluded user mobility or did not consider user mobility into their simulation. There is one work that considered users movement [8], where the macrocell users moved at a walking speed of $1 \mathrm{~m} / \mathrm{s}$. The work also introduced an idle mode for femtocells based on user activity detection.

Recently, the authors in [9] proposed a novel design of cellular networks, which is called "small-cell networks (SCNs)". SCNs are defined as cellular networks with very dense deployment of self-organizing, low-cost, low-power base stations. It is believed that SCNs are both cost and energy efficient solution to meet the forecasted traffic growth. However, it is also recognized that the realization of SCNs will encounter considerable challenges, especially because the conventional cellular networks have existed for a long time with enormous number of large-coverage base stations (macrocells) deployed all around the world. Since replacing those base stations with "small-cells" is supposed to be highly unlikely to take place in the near future, the possible target of SCNs would be the regions where cellular networks are not deployed yet.

\section{A Heterogeneous Cellular Network: Design under Test}

Network providers planning to expand their service in a new area should consider the base station deployment strategy in order to minimize the power consumption. There are some options, namely, (1) to employ a small number of macrocells with high transmission power, (2) to employ a large number of microcells with low transmission power, or (3) a combination of both creating a HTCN.

Since smaller cells consume less power, the deployment of smaller cells is encouraged with respect to energy efficiency. Typical coverage of a microcell is a few hundred meters. Smaller coverage in order of a few meters of indoor area can be achieved in femtocells. Usually, microcells are deployed in addition to the existing macrocells in the areas with high traffic, while femtocells are deployed in indoor areas to provide indoor $3 \mathrm{G}$ (3rd generation mobile telecommunications) coverage. According to the case study [1], femtocell deployment could result in 7:1 operational energy advantage ratio compared to macrocell networks for similar service.

In this paper, the HTCN contains macrocells, microcells and femtocells, with the topology as shown in Fig. 1(b). The HTCN is compared to HMCN with the topology as shown in Fig. 1(a). A network area can be divided into three kinds of regions: office and public places $(\mathrm{O})$, universities $(\mathrm{U})$, and residential $(\mathrm{R})$ as shown in Fig. 2. In the HTCN, microcells are deployed densely in the $\mathrm{O}$ regions and sparsely in the $\mathrm{U}$ 
regions, while femtocells are deployed distributively in the $\mathrm{R}$ regions. This consideration is taken to model real operation condition, where many mobile users exist at the same places at the same time.

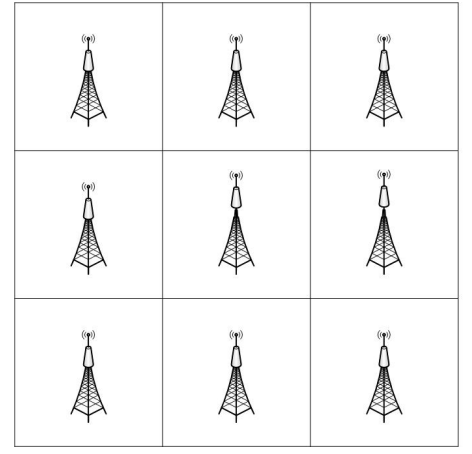

(a)

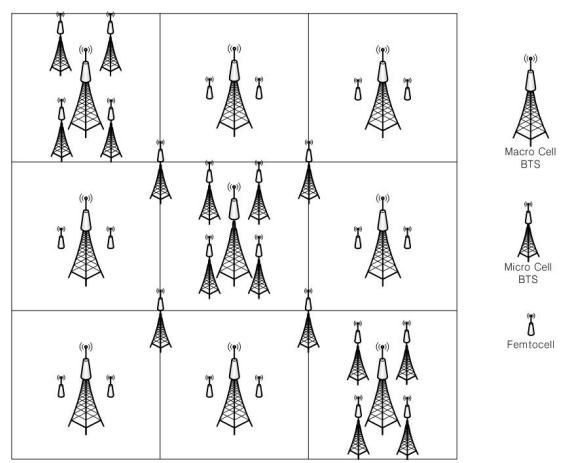

(b)

Fig. 1. (a) A homogeneous cellular network and (b) A heterogeneous cellular network

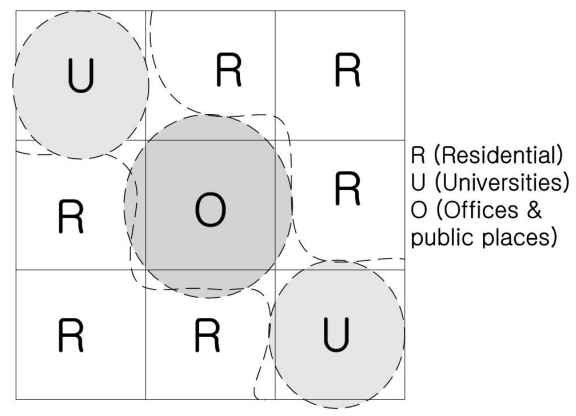

Fig. 2. Three kinds of regions in a network

A user mobility pattern is adapted on the basis of usual human mobility in daily life. By using this mobility pattern, the performance study is expected to be more realistic compared to random mobility pattern. Moreover, in HTCN, the users' location is especially significant to determine which base stations should provide service to certain users, and to clearly compare dense populated areas with scarce ones. In the proposed user mobility pattern, the overall population of mobile users is divided into 3 classes labeled A, B and C, and their mobility is defined according to certain time slots as follows (illustrated in Fig. 3):

- $40 \%$ of total population: users A. They start from area R, and then move towards area $\mathrm{O}$ and stay there or move around for some time. After that, some of them move around area $\mathrm{O}$ before move back to area $\mathrm{R}$ or directly return to area $\mathrm{R}$ and stay until the end of simulation time. Users A reflect office workers.

- 30\% of total population: users B. They start from area R, and then move towards area $\mathrm{U}$ and stay for some time (lower than users A stay time in area $\mathrm{O}$ ). After that, 
they move around area $\mathrm{O}$ before move back to area $\mathrm{R}$ or directly return to area $\mathrm{R}$ and stay until the end of simulation time. Users B reflect academics.

- $30 \%$ of total population: users C. Some of them merely stay in area R, and the rest of them move towards area $\mathrm{O}$ and then return to area $\mathrm{R}$ and stay until the end of simulation time. Users $\mathrm{C}$ reflect the rest of the population not belonging to class $\mathrm{A}$ or B, such as housewives.

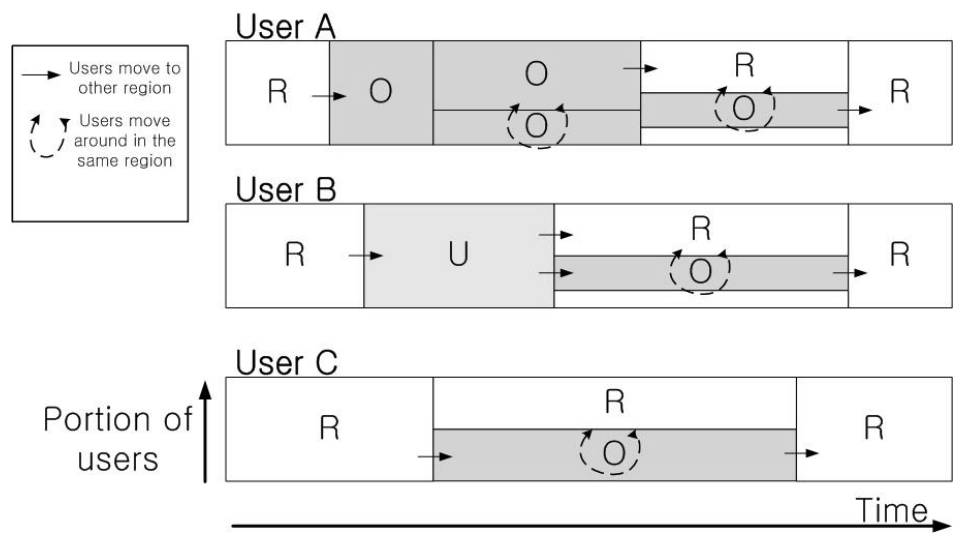

Fig. 3. User mobility pattern

\section{Performance Evaluation}

\subsection{Simulation Environment}

To compare the performance of HMCN and HTCN, a simulation is conducted with the settings as shown in Table 1. The HMCN contains macrocells and mobile users, while the HTCN contains all elements of macrocell, microcell, femtocell, and mobile users. The type of macrocell, microcell, and mobile nodes (handset) are the recent technology UMTS base stations, UMTS microcells, and UMTS/3G mobile phone, respectively, whereas the femtocells are assumed to be compatible with that technology. The numerical parameters are adapted from the reference papers.

All network elements, except macrocell, have sleep mode, which is to turn off some electronics equipments when there is no traffic to serve. Despite there are some works proposing idle/sleep mode for macrocells, the real adaptation of such a system is not exist yet. The microcells, femtocells, and mobile nodes are assumed to have idle/sleep mode by default. Therefore, macrocells consume power equal to its total power consumption $(1.85 \mathrm{KW})$ all the time. Based on the power consumption distribution in base stations [4], the proportion of power consumed in idle mode and sleep mode are defined. In the sleep mode, only the power supply is needed, which takes $7.5 \%$ of total power consumption. In the idle mode, air conditioning is turned on, which adds $17.5 \%$ of total power consumption, resulting in $25 \%$ of total power consumption. Both settings are applied for microcells and femtocells, while mobile nodes have only sleep mode which consumes $0.017 \mathrm{~W}$. 
Table 1. Simulation settings

\begin{tabular}{l|llll}
\hline \multirow{2}{*}{ Parameter } & HMCN & - & - & HMCN \\
& HTCN & HTCN & HTCN & HTCN \\
\hline \multirow{3}{*}{ Number of BS/users } & Macrocell & Microcell & Femtocell & Mobile users \\
Type & 9 & 16 & 12 & 90 \\
Transmission range & UMTS & UMTS & Femtocell & UMTS/3G \\
Idle mode \& sleep mode & $1000 \mathrm{~m}$ & $250 \mathrm{~m}$ & $10 \mathrm{~m}$ & - \\
Transmission power & No & Yes & Yes & Yes \\
Power consumption & $0.8 \mathrm{KW}$ & $10 \mathrm{~W}$ & $0.1 \mathrm{~W}$ & $0.25 \mathrm{~W}$ \\
Data rate & $1.85 \mathrm{KW}$ & $0.25 \mathrm{KW}$ & $7 \mathrm{~W}$ & $1 \mathrm{~W}$ \\
\hline Backbone & $384 \mathrm{Kbps}$ & $5 \mathrm{Mbps}$ & $14.4 \mathrm{Mbps}$ & - \\
\hline
\end{tabular}

The traffic is voice traffic [10] which divided into 2 types: normal traffic and femtocell traffic. The normal traffic is executed between random pairs of source and destination, whereas femtocell traffic is executed between source nodes that possess a femtocell to random destinations. In the normal traffic, the simulation time is divided into 3 phases. At the first and third phases, the traffic is low (10 sessions, i.e., 10 pairs of caller and receiver) and at the second phase, the traffic is high ( 35 sessions). These specifications are to model high traffic during the noon or working hours. The femtocell traffic is moderate (6 sessions out of 12 femtocells) and constant. In the simulation, both normal traffic and femtocell traffic are applied simultaneously. Our simulation is performed on Network Simulator 2 (NS-2) [11].

\subsection{Simulation Results and Discussion}

In our performance study, normalized power consumption, which is power consumed by each base station divided by its coverage area, is primarily taken into account. Throughput is also observed in terms of user data rate, and normalized power per throughput is evaluated and compared as a combined metric of power consumption and throughput.

Since the macrocells do not have neither idle mode nor sleep mode, one could predict that the HTCN, with more network elements added, would result in higher total power consumption. Fig. 4 shows the normalized power consumption of HMCN and HTCN. The total power consumption of HTCN is $905.16 \mathrm{~W} / \mathrm{Km}^{2}$, which is higher that of HMCN, 783.0 W/Km ${ }^{2}$. In case macrocells in both HMCN and HTCN do not have idle/sleep mode, apparently HTCN would consume higher total operational power since it has more network elements (microcells and femtocells). However, macrocells in HTCN has higher activity than the ones in $\mathrm{HMCN}$, showing higher utilization. Therefore, if macrocells have idle/sleep mode, HTCN might perform better energy conservation and utilization compared to HMCN.

Fig. 5 shows the throughput and drop rate of HTCN and HMCN. The throughput of HTCN is $12.5 \%$ higher and its drop rate is $7.5 \%$ lower than HMCN. If the normalized power consumption is divided by throughput, called normalized power per 
throughput, then $\mathrm{HTCN}$ outperforms HMCN. In HTCN, the normalized power per throughput is $139.81 \mathrm{~W} / \mathrm{Km}^{2} / \mathrm{Mbps}$, while it is $143,68 \mathrm{~W} / \mathrm{Km}^{2} / \mathrm{Mbps}$ in $\mathrm{HMCN}$. For that reason, HTCN is able to achieve higher data rate in energy-efficient manner. Moreover, the microcells and femtocells of HTCN are found to be underloaded. In dense traffic, HTCN is expected to achieve higher throughput than HMCN.

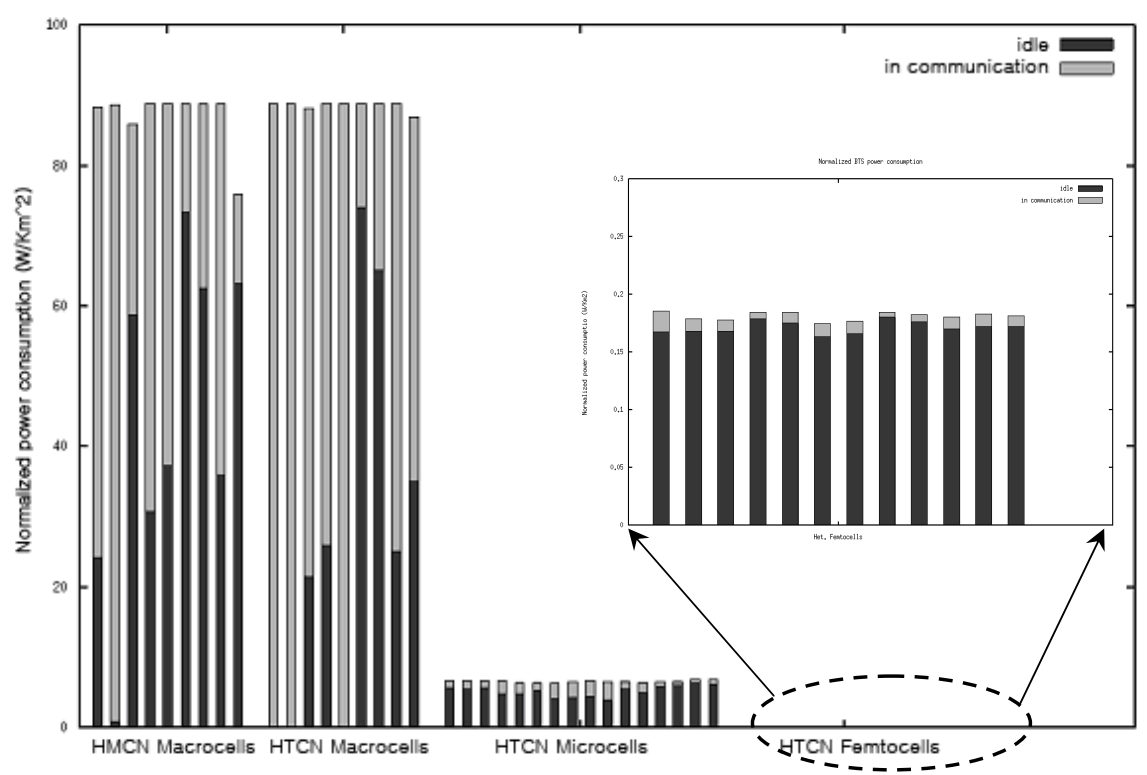

Fig. 4. Normalized power consumption of the whole base stations of HMCN and HTCN

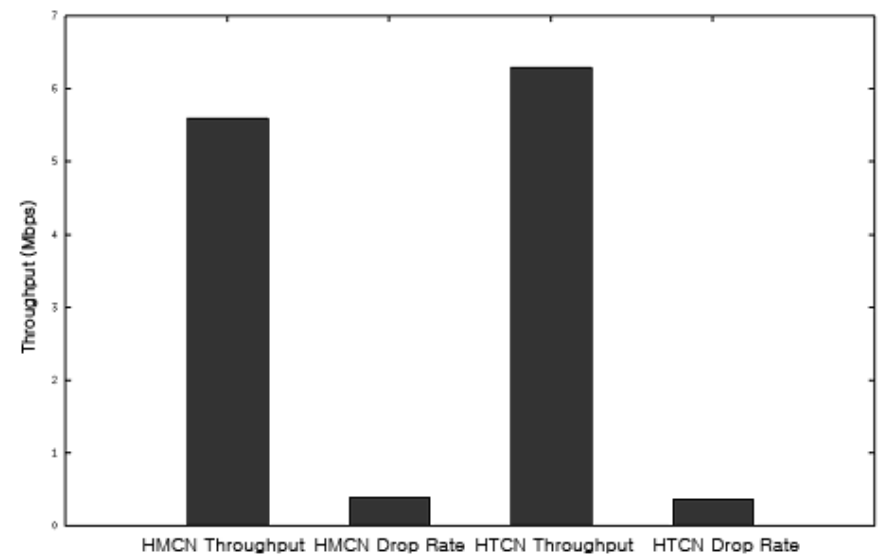

Fig. 5. Throughput and drop rate of $\mathrm{HMCN}$ and $\mathrm{HTCN}$ 


\section{Conclusions}

In this paper, we have studied the performance of HTCN and HMCN, in terms of power consumption and data rate (throughput). HTCN consists of macrocells, microcell and femtocells, whereas the HMCN consists of macrocells only. The network area is divided into three regions and a user mobility pattern is defined to model real mobile communication environment. The simulation results show that HTCN has the potency to support higher user data rate in energy-efficient manner. However, because the macrocells do not have idle/sleep mode, the power consumption of both cellular networks is relatively high. In the future, if macrocells have idle/sleep mode, HTCN is predicted to outperform HMCN in term of energy conservation. Moreover, the simulation on HTCN's microcells and femtocells are found to be underloaded. Thus, in dense traffic, HTCN is expected to achieve higher throughput and lower drop rate compared to HMCN.

\section{References}

1. Forster, C., Dickie, I., Maile, G., Smith, H., Crisp, M.: Understanding the Environmental Impact of Communication Systems. Plextex Final Report (2009)

2. Holma, H., Toskala, A.: LTE for UMTS - OFDMA and SC-FDMA Based Radio Access. John Wiley \& Sons Ltd., Chippenham (2009)

3. Arnold, O., Richter, F., Fettweis, G., Blume, O.: Power consumption modeling of different base station types in heterogeneous cellular networks. Future Network and Mobile Summit, 1-8, 16-18 (2010)

4. Hasan, Z., Boostanimehr, H., Bhargava, V.K.: Green Cellular Networks: A Survey, Some Research Issues and Challenges. IEEE Communications Surveys \& Tutorials, 524-540 (2011)

5. Claussen, H., Ho, L.T.W., Pivit, F.: Effects of Joint Macrocell and Residential Picocell Deployment on the Network Energy Efficiency. In: 19th IEEE International Symposium on Personal, Indoor and Mobile Radio Communications, pp. 15-18 (2008)

6. Chee, D., Kang, M., Lee, H., Jung, B.: A Study on the Green Cellular Network with Femtocells. In: Third International Conference on Ubiquitous and Future Networks (ICUFN), pp. 235-240 (2011)

7. Calin, D., Claussen, H., Uzunalioglu, H.: On Femto Deployment Architectures and Macrocell Offloading Benefits in Joint Macro-Femto Deployments. IEEE Communications Magazine 48, 26-32 (2010)

8. Ashraf, I., Ho, L.T.W., Claussen, H.: Improving Energy Efficiency of Femtocell Base Stations via User Activity Detection. In: IEEE Wireless Communications and Networking Conference (WCNC), pp. 1-5 (2010)

9. Hoydis, J., Kobayashi, M., Debbah, M.: Green Small-cell Networks. IEEE Vehicular Technology Magazine 6, 37-43 (2011)

10. Voice Over IP - Per Call Bandwidth Consumption (2012), http: / / www.cisco.com/en/US/tech/tk652/tk698/ technologies_tech_note09186a0080094ae2.shtml

11. The Network Simulator - ns-2 (2012), http: / / www . isi.edu/nsnam/ns / 